\title{
A survey of the status of nuclear cross section data for criticality safety
}

\author{
D.L. Smith ${ }^{\mathrm{a}, \mathrm{b}}$ \\ NE Division, Building 208, Argonne National Laboratory, 9700 South Cass Avenue, Argonne, IL 60439, USA
}

\begin{abstract}
Assurance of nuclear criticality safety is necessary to cultivate public acceptance of nuclear technology and nuclear power. Accurate nuclear data are needed in order to support the analytical studies required to achieve this goal. In the United States, the Nuclear Data Advisory Group (NDAG) is a multi-laboratory collaboration that has been organized by the US Department of Energy to accomplish this task. NDAG maintains an updated list of materials that are considered important for investigations of nuclear criticality safety. An independent survey of the current status of existing nuclear reaction data pertaining to these listed materials has been made with a particular emphasis on neutron cross sections. The results from this investigation are reported in this contribution.
\end{abstract}

\section{Introduction}

An important component of the US Department of Energy (US DOE) research activities directed toward promoting the safe use of nuclear power is the Nuclear Criticality Safety Program (NCSP). One of the tasks associated with this mission is to insure that good quality evaluated nuclear data are available for use in the wide variety of analyses that are being carried out to enhance criticality safety. The Nuclear Data Advisory Group (NDAG) is a multi-laboratory collaboration that has been organized to accomplish this task. NDAG maintains an updated list of materials and data needs considered important for investigations of nuclear criticality safety. The most recent documented list is included in a report from NDAG to USDOE dated August 2006 [1]. The elemental materials mentioned in this list (taken from Appendix D of the document) appear here in table 1 .

Although NDAG discusses the status of data for these materials during its quarterly meetings, this author (who is not a member of NDAG) felt that an independent survey of the situation concerning these data might also be beneficial to the criticality safety community. The present investigation was undertaken to fulfill this objective. The scope and depth of the present survey is necessarily limited by the time that has been available to devote to the project prior to this conference. Of course, these details are extremely important for assessing the adequacy of the criticality safety nuclear database. Consequently, the investigation is ongoing and further results will be reported in the future as the information becomes available. Five sources of compiled and documented information were examined during the course of this study: i) information available in various existing evaluated nuclear data libraries, especially the US library ENDF/B-VII.0 [2], ii) compiled experimental data in the exchange format EXFOR (hereafter referred to simply as EXFOR) [2], iii) the Nuclear Wallet Cards [3], iv) the Atlas of Neutron Resonances [4] (hereafter referred to simply as Atlas), and v) Table of Isotopes, 8th Edition [5].

\footnotetext{
a Present author, e-mail: Donald.L.Smith@anl.gov

$b$ Present address: 1710 Avenida del Mundo \#1506, Coronado, CA 92118, USA
}

Table 1. List of the 49 elemental materials considered by NDAG to be important for the US Criticality Safety Program.

\begin{tabular}{|c|}
\hline Alphabetical List of Elemental Materials \\
\hline Aluminum, Americium, Berkelium, Beryllium, Bismuth, \\
Boron, Californium, Calcium, Carbon, Cerium, \\
Cesium, Chlorine, Chromium, Copper, \\
Curium, Europium, Fluorine, Gadolinium, \\
Hafnium, Hydrogen, Iron, Lead, Lithium, Magnesium, \\
Manganese, Molybdenum, Neodymium, \\
Neptunium, Nickel, Niobium, Nitrogen, Oxygen, \\
Plutonium, Potassium, Protactinium, Rhenium, Rhodium, \\
Rubidium, Samarium, Silicon, Sodium, Tantalum, \\
Technetium, Thorium, Titanium, \\
Tungsten, Uranium, Vanadium, Zirconium \\
\hline
\end{tabular}

\section{Survey procedures and scope}

This survey generated far more material than could be included in this written paper or in the poster presented at the conference. Therefore, the following approach has been taken to document this material [6]: a detailed report (hereafter referred to as "Report") is being prepared. It will document a number of specific comments about the various aspects of this survey that are too extensive to be included here. This material will become available in late 2007 from Argonne National Laboratory (ANL) at the Internet site www.ne.anl.gov/capabilities/nd/reports/ANLNDM161.html.

A collection of 830 individual plots of evaluated and experimental cross sections were generated by using an online plotting utility available from the National Nuclear Data Center at Brookhaven National Laboratory [2]. They were downloaded from the Internet in the *.gif format. These have been collected in a single file folder labelled "Plots" that will be posted at the ANL website. All available evaluations (not just ENDF/B-VII.0) from the file category MF $=3$ are shown along with EXFOR experimental data. The evaluated curves shown on these plots for energies in the resonance region and below are generally misleading since cross sections for this region are usually constructed directly by processing codes, primarily from archived resonance parameters with 
some numerical adjustments (as shown on the plots) that are taken from the $\mathrm{MF}=3$ files. Certain numerical as well as visual information has been assembled in an EXCEL spreadsheet (hereafter referred to as "Spreadsheet") that includes seven distinct worksheets. It too will be posted at the ANL website. "Spreadsheet" is composed of the following worksheets with the indicated titles: Worksheet 1 ("Inventory of Materials"), Worksheet 2 ("Reaction Energetics"), Worksheet 3 ("Cross Section Libraries"), Worksheet 4 ("Thermal \& Resonance Info"), Worksheet 5 ("ENDFBVII.0 Cross Sections"), Worksheet 6 ("ENDFB-VII.0 Neutron Emission"), and Worksheet 7 ("ENDFB-VII.0 Nu-bar"). The contents of these individual worksheets in "Spreadsheet" are discussed separately below by specific worksheet name.

"Inventory of Materials": Working from the elements appearing in table 1, a list (hereafter referred to as "Master List") has been prepared of the stable isotopes and their abundances as well as many important longer-lived isotopes, especially those for the heavier elements. Half lives were also tabulated where relevant. Isotopic abundances are important in assessing the relative importance of the isotopes in most instances, especially for cross sections in the fast-neutron region. However, in the resonance region even relatively minor isotopes can play a very significant role when they possess some very strong resonances.

"Reaction Energetics": The (n, $\gamma$ ) Q-values for all isotopes in "Master List" were computed using the Los Alamos National Laboratory on-line calculator Q-tool (available at t2.lanl.gov) and tabulated in this worksheet. Excitation energies of the first-excited states of these isotopes were also listed since this is important for assessing the influence of neutron inelastic scattering. Finally, laboratory threshold energies were calculated and tabulated for the following reaction processes since they are the ones that generally need to be considered for neutron energies $\leq 20 \mathrm{MeV}$ : $(n, p),(n, d),(n, t),\left(n,{ }^{3} \mathrm{He}\right)$, $(\mathrm{n}, \alpha),(\mathrm{n}, 2 \mathrm{n}),(\mathrm{n}, \mathrm{np}),(\mathrm{n}, \mathrm{nd}),(\mathrm{n}, \mathrm{n} \alpha)$, and $(\mathrm{n}, 3 \mathrm{n})$. Although for criticality safety concerns one is generally interested in the lower-energy region, evaluated nuclear data libraries typically extend from thermal to $20 \mathrm{MeV}$.

"Cross Section Libraries": All of the contemporary evaluated nuclear data libraries (ENDF/B-VII.0, JEFF-3.1, JENDL-3.3, BROND-2.2, and CENDL-2) were surveyed to determine which of them contained complete evaluations for isotopes in "Master List". Cells in this "Spreadsheet" worksheet were filled in light green when an evaluation was present and with pink if no evaluation was found in the library for a particular isotope. In addition, dates when the individual evaluated files were produced are indicated in those cells corresponding to ENDF/B-VII.0 (including the dates of any major revisions). Whenever an evaluation was found to predate 1990, the corresponding date was visually tagged by using red font. The intent of this tagging is to provide a clear indication of the extent to which relatively "old" evaluations can still be found in ENDF/B-VII.0.

"Thermal \& Resonance Info": The only library surveyed for the status of neutron thermal and resonance information was ENDF/B-VII.0. But, this information was supplemented by considering the contents of Atlas [4]. This "Spreadsheet" worksheet notes when information on thermal cross sections and resonance parameters is available for the isotope in question by filling the cell in question light green. When none is available, the cell is crossed out. Notice is made as to whether resonance information $(\mathrm{MF}=2$ and $\mathrm{MT}=151)$ is available in ENDF/B-VII.0 in a form that is directly accessible to processing codes. The information extracted from Atlas is grouped in this "Spreadsheet" worksheet according to the categories thermal cross sections $\left(\sigma_{\mathrm{s}}, \sigma_{\gamma}, \sigma_{\mathrm{p}}, \sigma_{\alpha}\right.$, and $\left.\sigma_{\mathrm{f}}\right)$, resonance integrals $\left(\mathrm{I}_{\mathrm{a}}, \mathrm{I}_{\gamma}, \mathrm{I}_{\mathrm{p}}\right.$, and $\left.\mathrm{I}_{\mathrm{f}}\right)$, and resonance widths $\left(\Gamma, \Gamma_{\mathrm{n}}, \Gamma_{\mathrm{n}}, \Gamma_{\gamma}, \Gamma_{\mathrm{p}}, \Gamma_{\alpha}, \Gamma_{\mathrm{f}}, \Gamma_{\mathrm{n}} \Gamma_{\gamma} / \Gamma\right)$. Each of these parameters is defined in the worksheet. A code is used in each cell to denote the extent to which uncertainty information for the values provided exists in Atlas. The code "E" indicates that uncertainties are provided for the thermal cross section and/or most of the listed resonance parameters in that category. On the other hand, "e" denotes (only for the resonance information) that the uncertainty information is fairly limited in that category. Finally, a blank (no code symbol) signifies that no resonance parameter uncertainty information is available from Atlas in that category.

"ENDFB-VII.0 Cross Sections": This "Spreadsheet" worksheet indicates which of the neutron reaction cross sections $(\mathrm{MF}=3)$ can be found in ENDF/B-VII.0 for each isotope from "Master List". The reaction processes included in the survey are: tot (total cross section), el (elastic scattering), inl (inelastic scattering), (n, $\gamma),(\mathrm{n}, \mathrm{p}),(\mathrm{n}, \mathrm{d}),(\mathrm{n}, \mathrm{t}),\left(\mathrm{n},{ }^{3} \mathrm{He}\right),(\mathrm{n}, \alpha)$, $(n, 2 n),(n, n p),(n, n d),(n, n \alpha),(n, 3 n)$, and $(n, f)$. Codes are again used in the corresponding cells to denote the status of this information. If the cell is crossed out, no evaluation exists for this process in ENDF/B-VII.0. Light green filling indicates that an evaluation exists but there are no corresponding EXFOR experimental data. Thus, the evaluation is based on nuclear modelling alone. Darker green filling indicates that both an evaluation and experimental data can be found. The extent of these data is not indicated so there could be anywhere from one to very many points. As mentioned above, whenever experimental data are available they are included in the plots along with the evaluated results from all relevant libraries. Finally, "COV" indicates that a corresponding covariance matrix exists for the ENDF/B-VII.0 evaluation in question.

"ENDFB-VII.0 Neutron Emission": There are three categories of evaluated neutron emission data to consider: Emission angular distributions ( $\mathrm{MF}=4)$, emission energy distributions $(\mathrm{MF}=5)$, and emission energy and angular distributions $(\mathrm{MF}=6)$. This "Spreadsheet" worksheet identifies which "Master List" isotopes are represented in ENDF/B-VII.0 for each category. If a particular MF category is represented, the corresponding cell is filled in light green. If it is not represented, the cell is crossed out.

"ENDFB-VII.0 Nu-bar": Nu-bar (neutrons emitted per fission) is categorized in evaluated files in three ways: Nu-bar total (MT = 452), Nu-bar delayed $(\mathrm{MT}=455)$, and Nu-bar prompt $(\mathrm{MT}=456)$. This "Spreadsheet" worksheet indicates the status of nu-bar information for fissionable actinide nuclei found in "Master List". If a particular MT category is represented, the corresponding cell is filled in light green. If it is not represented, the cell is crossed out. In some cases covariance information is available. This is indicated by the code "COV" found in the relevant cell. 


\section{Comments on the results from this survey}

Naturally, the question that interests most criticality safety analysts is: "Are the existing evaluated data (especially in ENDF/B-VII.0) adequate in scope and accuracy to meet our needs?" There are two requisites to be able to answer this question definitively. First, the accuracies of the various required evaluated nuclear data have to be specified (specifically, in the form of covariance matrices). Second, the sensitivities of the computed criticality parameters to these data need to be determined. Unfortunately, neither the data accuracies nor the system sensitivities are very well characterized in most cases. Covariance information is quite limited in ENDF/B-VII.0, although progress is being made in generating additional covariances to be included in future updates to the library. Sensitivity information is also lacking, largely because software adequate to perform such analyses is not yet widely available. So, this study, by necessity, is confined to indicating what does and does not exist and to making some very qualitative comments about uncertainties.

Neutron total, elastic, and inelastic scattering cross sections as well as radiative capture and fission cross sections (where applicable) are the most likely to be influential in criticality safety analyses. Charged-particle emission cross sections, e.g., (n,p), (n,d), etc., and multiple neutron emission processes such as $(n, 2 n)$ and $(n, 3 n)$ tend to have thresholds well above the energy region of greatest impact for criticality safety. Nevertheless, these reactions are included in the worksheet "ENDFB-VII.0 Cross Sections" for completeness.

The present conference paper, owing to the imposed page limitations, is restricted to offering some general observations on this topic based on the information that has been collected during the present survey. These comments are presented below. As mentioned above, more detailed commentary on individual isotopes, reactions, and physical parameters will appear in a future laboratory report [6].

Evaluated cross section data libraries: The worksheet "Cross Section Libraries" offers a visual impression of the relevant content of the five major nuclear data libraries. It is clear that ENDF/B-VII.0 is the most comprehensive of these. It is also the most up-to-date. JEFF-3.1 and JENDL-3.3 lag somewhat behind in content and they are also somewhat older, but not by a great deal in either respect. Considerably behind the leaders in both content, and older as well, are BROND-2.2 and CENDL-2. It should be pointed out that there is considerable commonality in the content of these libraries. They are far from being truly independent of each other.

Thermal cross sections: For the most part, the thermal scattering $\left(\sigma_{\mathrm{s}}\right)$, radiative capture $\left(\sigma_{\gamma}\right)$, and fission $\left(\sigma_{\mathrm{f}}\right.$, where relevant) cross sections for isotopes in "Master List" are reasonably well represented (both values and uncertainties), based on the evaluated files as well as Atlas. Furthermore, the accuracy is generally quite good. Therefore, the currently available information probably addresses the most important physical processes for criticality safety. However, large gaps in the database are observed for the other thermal cross sections. Thus, whether in the balance the available data are adequate remains to be determined, as suggested above.

Resonance integrals: Radiative capture $\left(\mathrm{I}_{\gamma}\right)$ and fission $\left(\mathrm{I}_{\mathrm{f}}\right.$, where relevant) resonance integral data and uncertainties are available for most isotopes in "Master List", according to Atlas. Otherwise, the evaluated results in this category are relatively spotty. In spite of this, the information that is currently available probably addresses the most importance concerns for criticality safety.

Resonance widths: Neutron $\left(\Gamma_{\mathrm{n}}\right)$, gamma $\left(\Gamma_{\gamma}\right)$, and fission $\left(\Gamma_{\mathrm{f}}\right.$, where relevant) width values and corresponding uncertainties appear to be fairly well represented for the isotopes from "Master List". However, it should be noted that there tend to be as many "e" (limited uncertainty information) code entries as "E" (extensive uncertainty information) code entries in the cells of the "Thermal \& Resonance Info" worksheet of "Spreadsheet". This suggests that there is definitely room for improvements in the database. High quality resonance information can be obtained best from direct measurements of the important neutron radiative capture and fission processes in the resonance region.

Total cross sections: Evaluated neutron total cross sections are available for most isotopes from "Master List". In the resonance region, these are usually computed from scattering and absorption resonance parameters. In the fast-neutron region, many of these data sets are derived from nuclear optical-model calculations since measurements tend to be limited to natural (elemental) materials and a few of the more common isotopic materials. These calculations tend to be fairly reliable because the variations with energy and isotope tend to be both modest and systematic in nature. This is supported by the fact that the curves from various libraries (when independent) tend to show relatively modest spreads.

Neutron elastic and inelastic scattering: In principle, the elastic scattering cross section (both shape and compound components) can be calculated from a given set of optical model parameters. Elastic experimental data are available for quite a few elemental materials and in some cases for isotopic materials that are available in sufficient quantities to prepare scattering samples. However, in evaluations the elastic scattering cross sections are typically determined by subtracting the sum of reaction cross sections from the total cross section, thereby achieving internal numerical consistency of the file. In cases where EXFOR data are available, the various libraries tend to agree reasonably well. Otherwise, they vary from fairly good agreement to relatively poor agreement between the various libraries. In the energy region of greatest interest for criticality safety, inelastic scattering is generally dominated by the lowest excited states of the target nucleus where the spins and parities are generally well known. In principle it should be possible to calculate these cross sections with reasonably good accuracy. In practice, the model-calculated results vary considerably. This issue is of particular concern for the actinides where the neutronics can be strongly influenced by excited states located at relatively low excitation energies.

Neutron fission: Fission cross sections for major actinides are generally well known over a wide energy range. The gaps in fission data are to be found in the minor actinides where it is acknowledged that much more work is needed. These data must be acquired from measurements since no fission models are able to provide results with sufficient accuracy. Many of the remaining needed measurements will be difficult because of sample and radioactivity issues, e.g., spontaneous fission. 
Neutron radiative capture: For criticality safety concerns, this process is most strongly affected by the resonance region. Since the properties of resonances vary so markedly, and in an unsystematic way, with both isotope and energy, it is difficult to draw general conclusions. The situation must be examined isotope by isotope. It is evident from "Spreadsheet" that the capture resonance information is quite extensive for the major actinides but less so for minor actinides. The situation for fission products, structural materials, and "poisons" is quite mixed. Since there are no comprehensive theories that allow for prediction of individual resonance properties, they must be measured. Sample limitations (abundance, chemical stability, etc.) are important considerations in establishing what can be measured and the attainable accuracies. Measurements in the fast-neutron region are difficult, even when abundant sample material is available, because of the interfering effects of lowenergy background neutrons. Radiative capture cross sections are also difficult to calculate in the fast-neutron region. As a consequence, differences in the evaluated cross sections in this region between the various independent libraries often amount to several orders of magnitude.

Charged-particle emission cross sections: As mentioned earlier, cross sections for reactions such as $(n, p),(n, d),(n, t)$, $\left(\mathrm{n},{ }^{3} \mathrm{He}\right),(\mathrm{n}, \alpha),(\mathrm{n}, \mathrm{np}),(\mathrm{n}, \mathrm{nd})$, and $(\mathrm{n}, \mathrm{n} \alpha)$ tend to have thresholds in the few to several $\mathrm{MeV}$ range, and thus are of relatively minor importance for criticality safety applications. These cross sections can be calculated using nuclear models, but with relatively poor accuracy. In the absence of experimental data, differences between libraries ranging from a few percent to an order of magnitude or more are common. When good quality data are available, usually from activation measurements, the agreement is much better.

$(\mathrm{n}, 2 \mathrm{n})$ and $(\mathrm{n}, 3 \mathrm{n})$ reactions: These cross sections inevitably exhibit thresholds in the several $\mathrm{MeV}$ range up to well beyond $10 \mathrm{MeV}$ thereby virtually insuring that they will be of minor importance for criticality safety. The $(\mathrm{n}, 2 \mathrm{n})$ cross section is large and can generally be calculated with reasonably good accuracy. The results from most libraries will tend to agree to within 10-20\% except in the near threshold region. The agreement can be even better when good quality experimental data are available to guide the evaluations. Again, these data are usually obtained from activation measurements, but a few good quality direct neutron-emission measurements have been reported. Cross sections for $(n, 3 n)$ are more poorly known than $(\mathrm{n}, 2 \mathrm{n})$, but they are unimportant for criticality safety applications owing to the very high reaction thresholds (always above $10 \mathrm{MeV}$ ).

Neutron emission: Measurements of neutron emission other than fission (both angular and energy) tended to be relatively spotty across the Periodic Table, and are frequently limited in their energy range. In fact, many of the measurements have been performed in the vicinity of $14 \mathrm{MeV}$ using elemental sample materials. These data have been useful for the development of compound and pre-compound nuclear models, but they have limited use for criticality safety applications. Generally, evaluated neutron-emission results reported in evaluated cross-section libraries are obtained from nuclear modelling. Evaluated angular emission (MF = 4) information is available in ENDF/B-VII.0 for all "Master List" isotopes, but this is not the case for energy $(\mathrm{MF}=5)$ or energy-angular $(\mathrm{MF}=6)$ emission. Whether the available results are adequate or not for criticality safety remains to be determined from sensitivity studies. Clearly covariance information, which is universally missing for neutron emission, will eventually be required for this purpose.

Nu-bar: Knowledge of the number of neutrons emitted per $\overline{\text { fission }}$ - total $(\mathrm{MT}=452)$, delayed $(\mathrm{MT}=455)$, and prompt $(\mathrm{MT}=456)$ - is clearly of exceedingly high importance for criticality safety studies. With few exceptions (to be found among the minor actinides) quite accurate nu-bar information is available in ENDF/B-VII.0. This information is based on extensive measurements compiled and evaluated over the course of many decades. In the case of ${ }^{232} \mathrm{Th}$ and ${ }^{235} \mathrm{U}$, some covariance information is also available. Covariance information should be generated for all nu-bar values, and the remaining gaps in the data for minor actinides need to be filled. Otherwise, it is likely that the existing information is sufficient for most criticality safety applications.

The author is indebted to the US Department of Energy for the financial support that enabled him to attend the present conference.

\section{References}

1. Nuclear Data Advisory Group, Nuclear Criticality Safety Program Five-Year Plan, Report to the US Department of Energy, August 2006, unpublished.

2. National Nuclear Data Center (NNDC), Brookhaven National Laboratory (www.nndc.bnl.gov).

3. J.K. Tuli, Nuclear Wallet Cards (National Nuclear Data Center, Brookhaven National Laboratory, 2005).

4. S.F. Mughabghab, Atlas of Neutron Resonance, 5th edn. (Elsevier Publishing Company, Amsterdam, 2006).

5. R.B. Firestone et al., Table of Isotopes, 8th edn. (John Wiley \& Sons, Inc., New York, 1996).

6. D.L. Smith, Report ANL/NDM-165, Argonne National Laboratory (to be posted on the ANL/NDM website in 2007, www.ne.anl.gov/capabilities/nd/reports/ANLNDM161.html). 